\title{
nuevo edificio en la fábrica Deckel, en Munich alemania
}

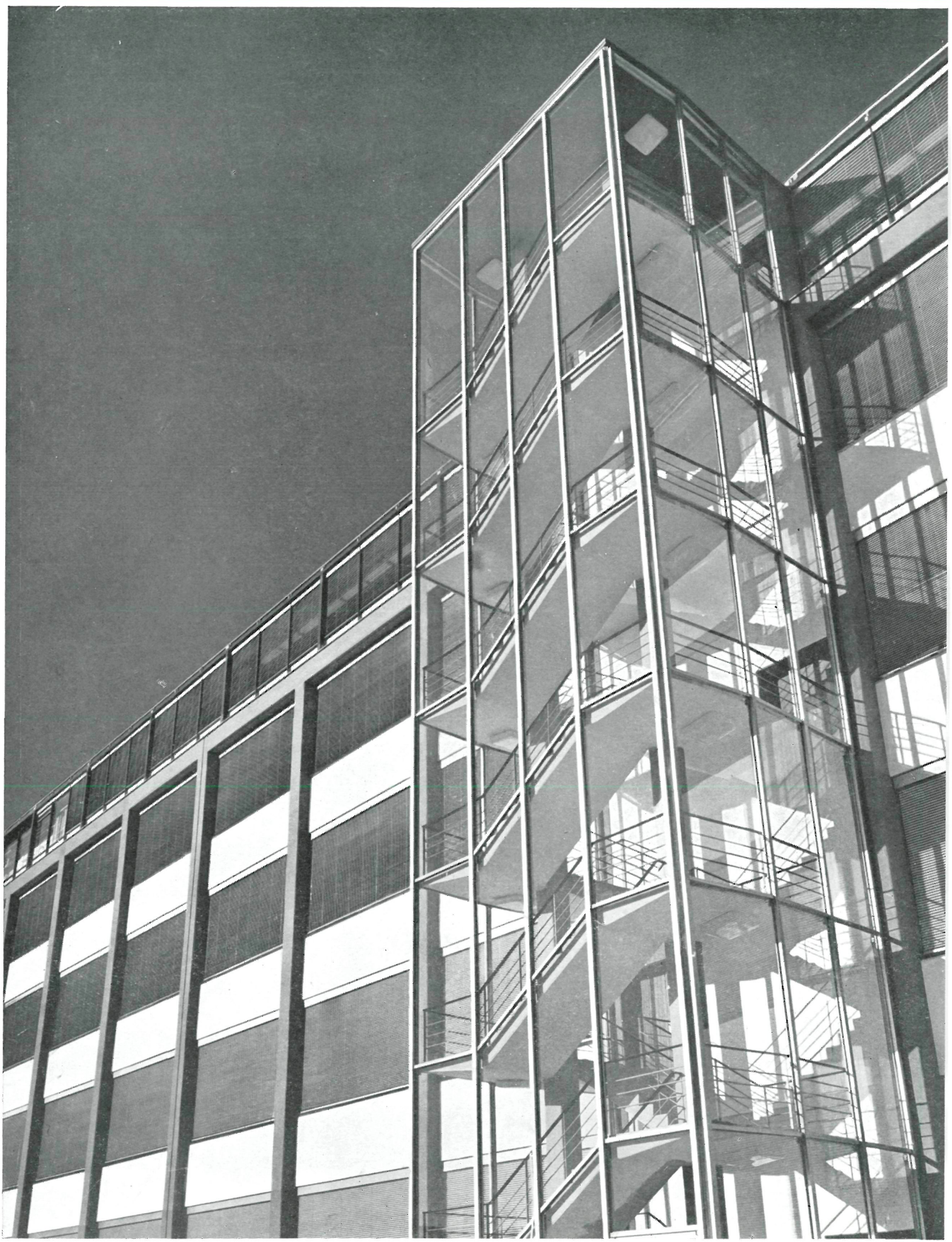




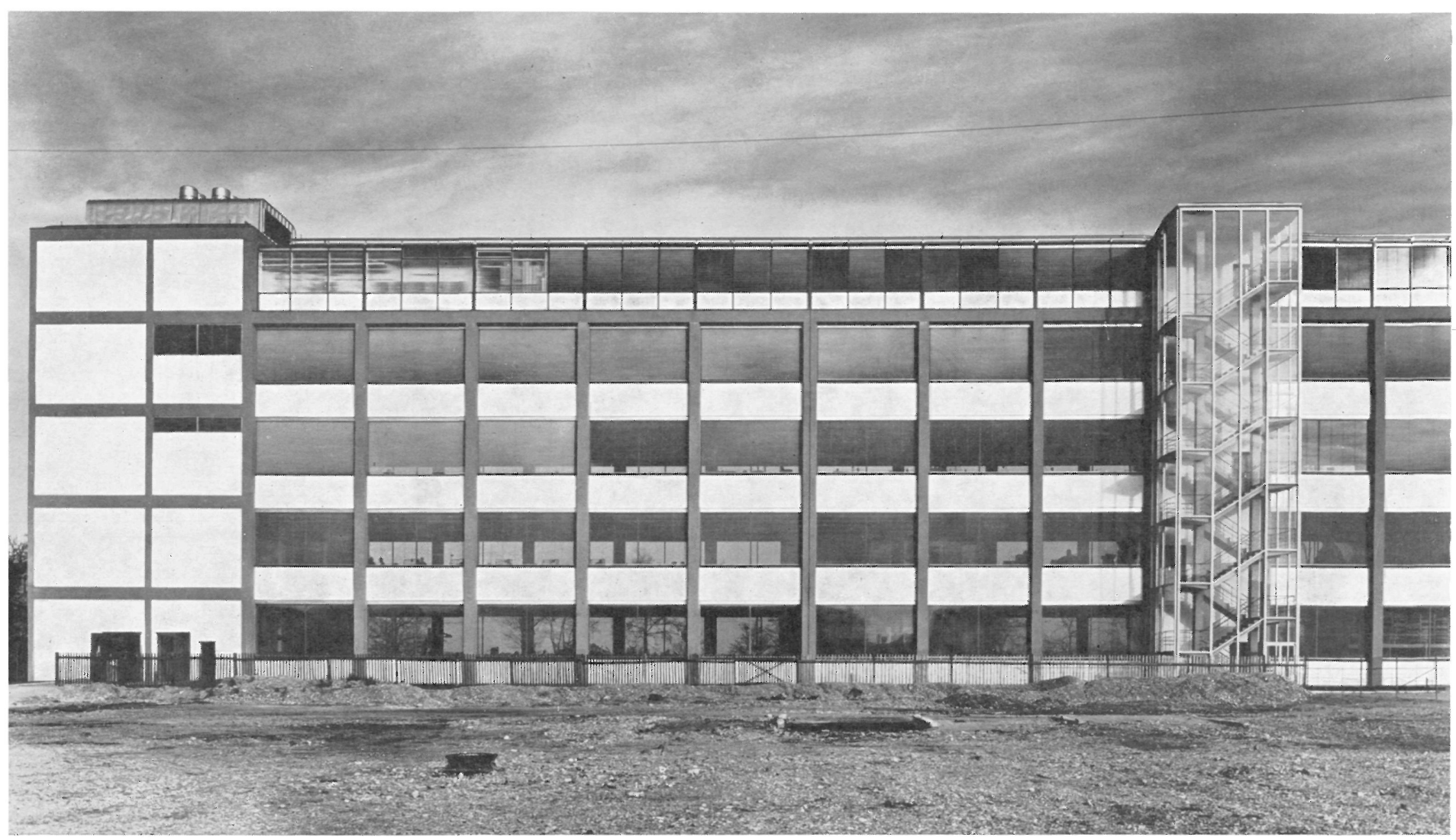

simopsis

Destinado a la fabricación de accesorios, este edificio consta de: sótano, planta baja y cuatro plantas de altura.

En la planta de sótano se encuentra una de las centrales de climatización, vestuarios, aseos, duchas del personal, etc. Las otras plantas están dedicadas a la fabricación; finalmente, la mayor parte de la superficie de la planta superior está ocupada por las oficinas y los locales sociales.

El edificio es claro, sencillo y funcional y está dotado de acondicionamiento e iluminación inmejorables.

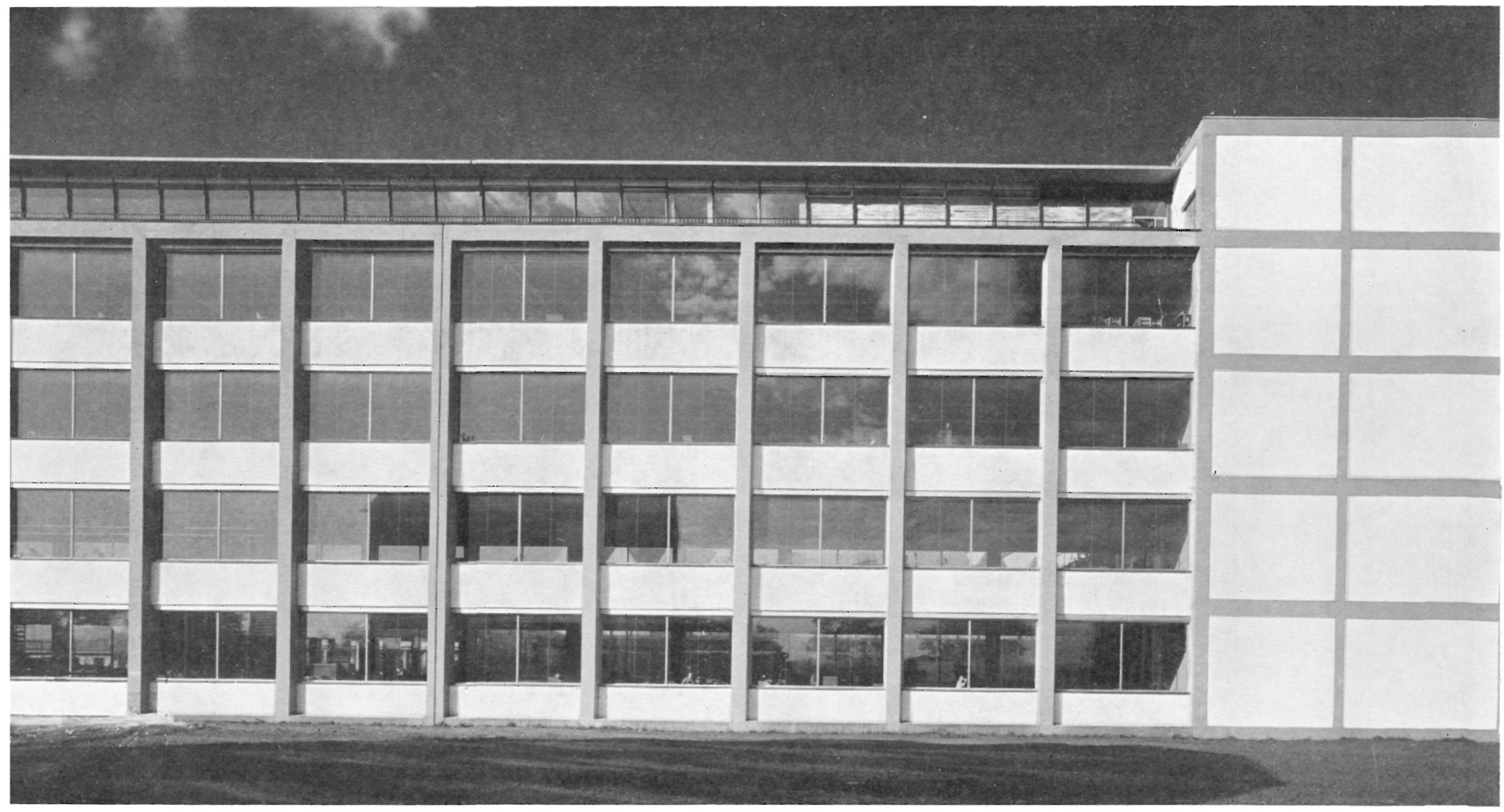


La firma «Friedrich Deckel», fabricante de maquinaria, ha erigido, en sus terrenos de MunichObersendling, una serie de nuevas construcciones; entre ellas figura este edificio dedicado a la fabricación de accesorios.

La citada edificación ha sido desarrollada en altura, debido al hecho de que el solar disponible era de superficie muy limitada, lo que forzó a organizar la superficie productiva en varios pisos.

El bloque tiene una longitud de $130 \mathrm{~m}$, y consta de sótano, planta baja y cuatro plantas de altura; las plantas destinadas a fabricación, de $100 \mathrm{~m}$ de longitud y unos $15 \mathrm{~m}$ de anchura, fueron proyectadas libres de obstáculos constructivos con objeto de facilitar el montaje de las máquinas.

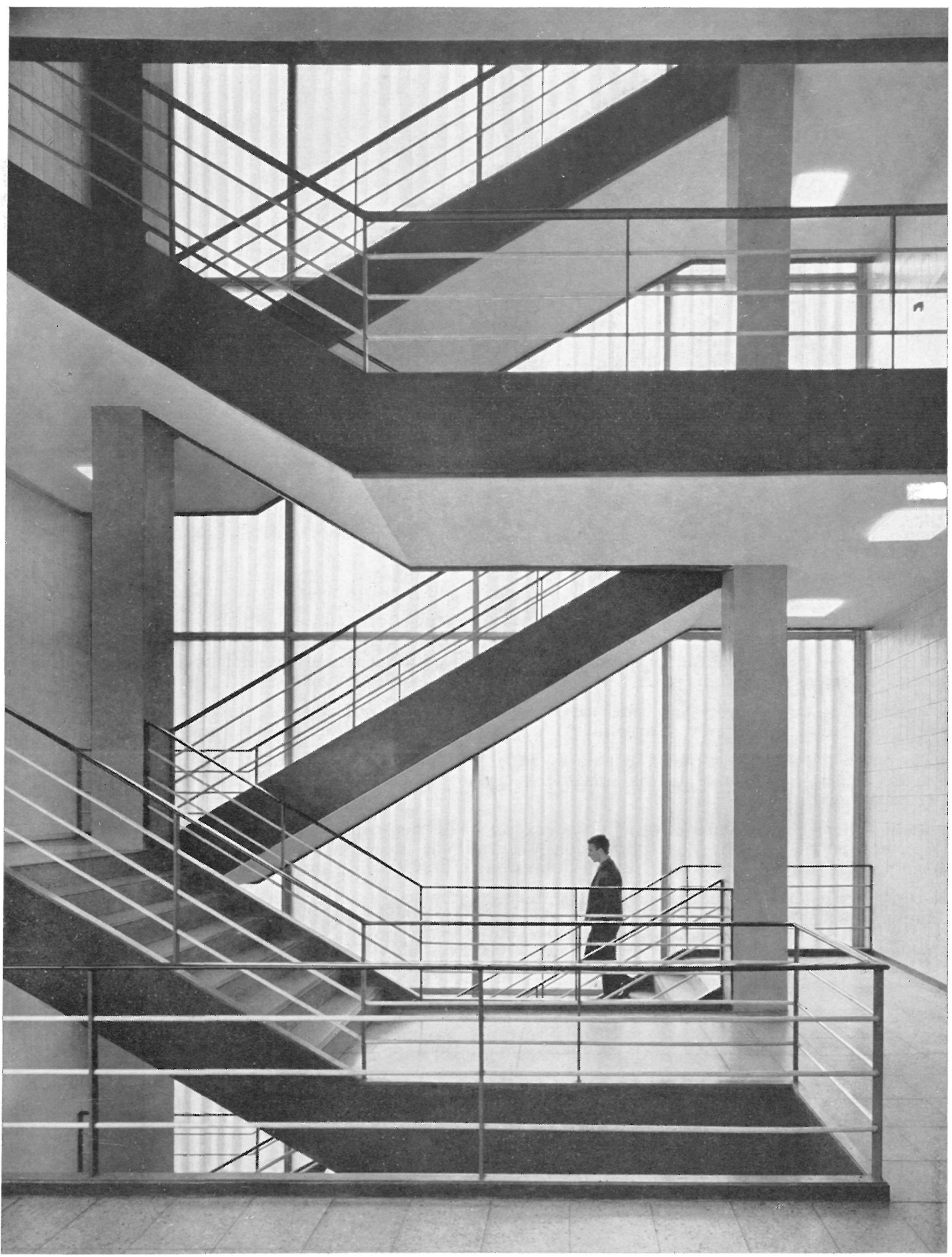




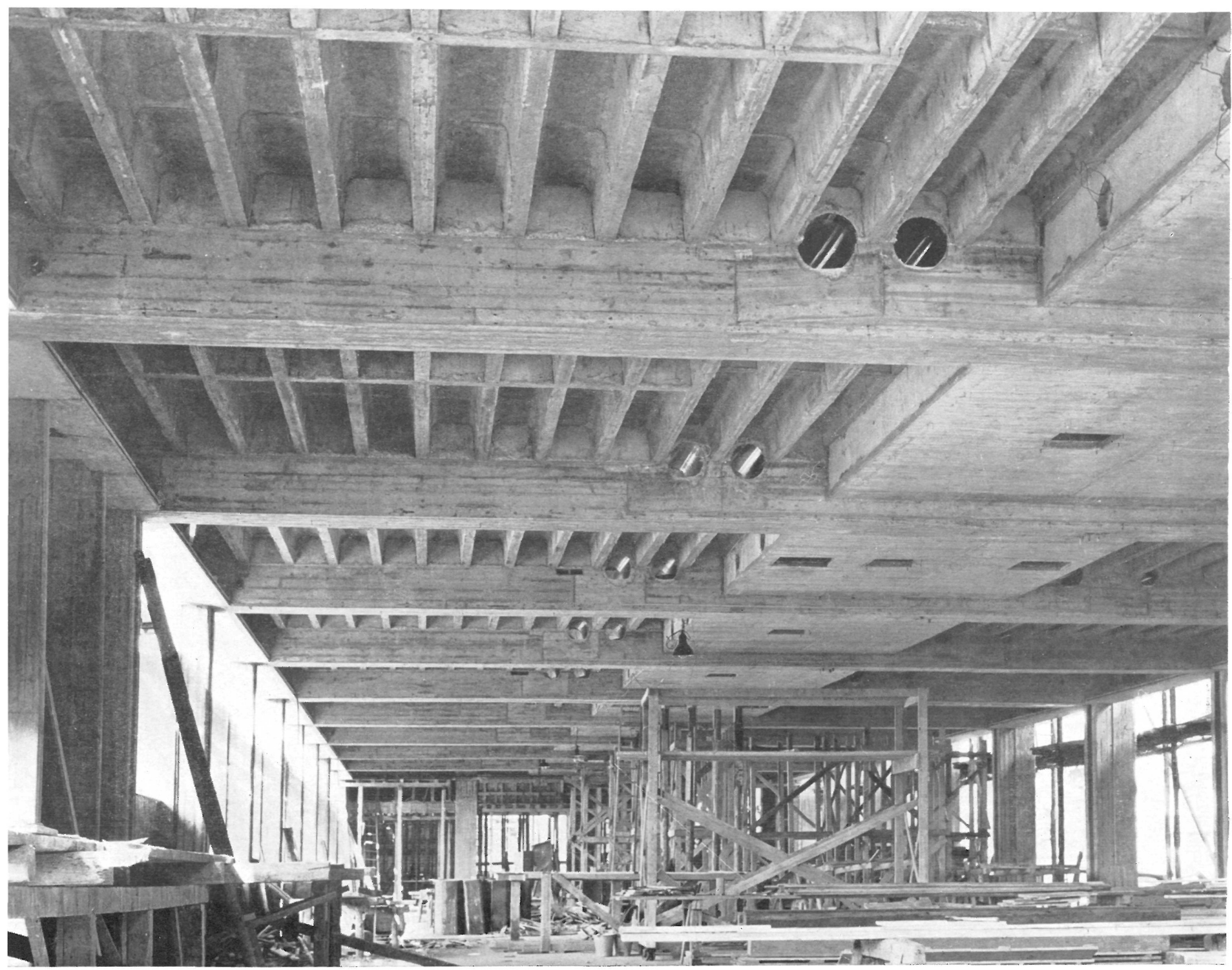

Fotos: H. HEIDERSBERGER

En los testeros de la edificación están agrupadas las comunicaciones verticales y servicios generales: ascensores, escaleras, servicios, aseos y conductos de las instalaciones.

Los núcleos de fabricación han sido distribuidos de forma que, además de posibilitar la circulación de carretillas eléctricas, permiten ampliar la superficie de producción en caso necesario.

En el centro del edificio y adosado a la fachada hay un prisma acristalado que contiene la escalera para casos de incendio, además de las mangueras para el agua, de extinción, e instalaciones de la fábrica y primeros auxilios.

Como la fabricación de maquinaria de precisión exige determinados requisitos en las condiciones de trabajo, y equipo de las naves de fabricación, este edificio presenta amplias superficies acristaladas en los dos muros longitudinales, lo que proporciona una iluminación óptima en el interior.

Las ventanas están acristaladas con vidrio termopán y van equipadas con celosías exteriores de láminas de metal ligero, accionadas individualmente mediante dispositivos eléctricos.

Debido a la orientación O.-E. del edificio, se origina una fuerte y prolongada radiación solar, que sobre todo en verano, junto con el calor producido por las máquinas, podría originar un recalentamiento insoportable de las naves.

Por este motivo se dotó de aire acondicionado, con regulación totalmente automática, a toda la zona productiva del edificio; en este aspecto, es asimismo importante: el control de la humedad, para reducir o anular totalmente la corrosión de las piezas en fabricación.

La distribución general del edificio es la siguiente: en el sótano se encuentra la central de climatización para la planta baja y el primer piso; los vestuarios, aseos y duchas para 500 empleados, etc. Una escalera exterior, que puede calentarse en invierno para mantenerla sin nieve ni hielo, constituye el paso de los trabajadores a los locales comunitarios del sótano. 
तล

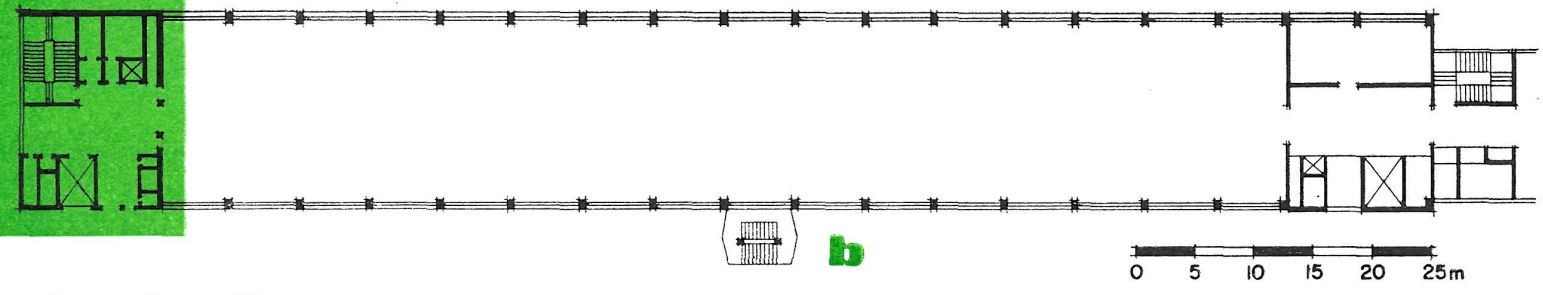

planta trapo

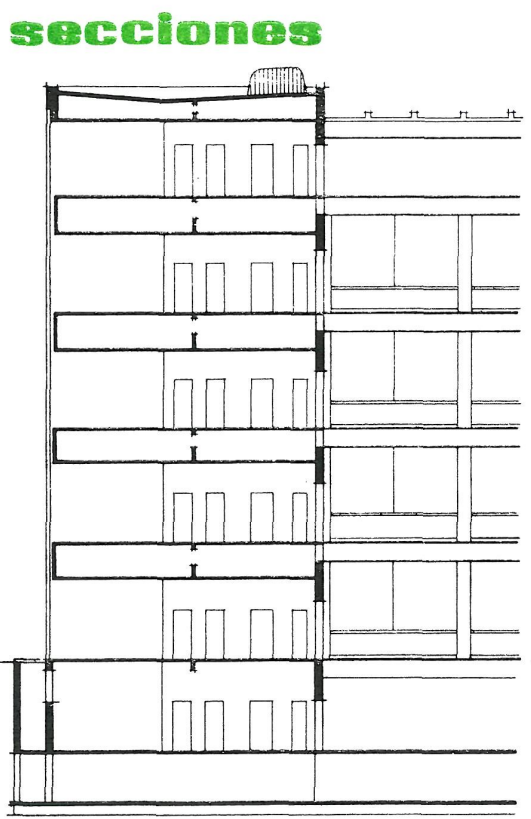

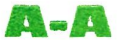

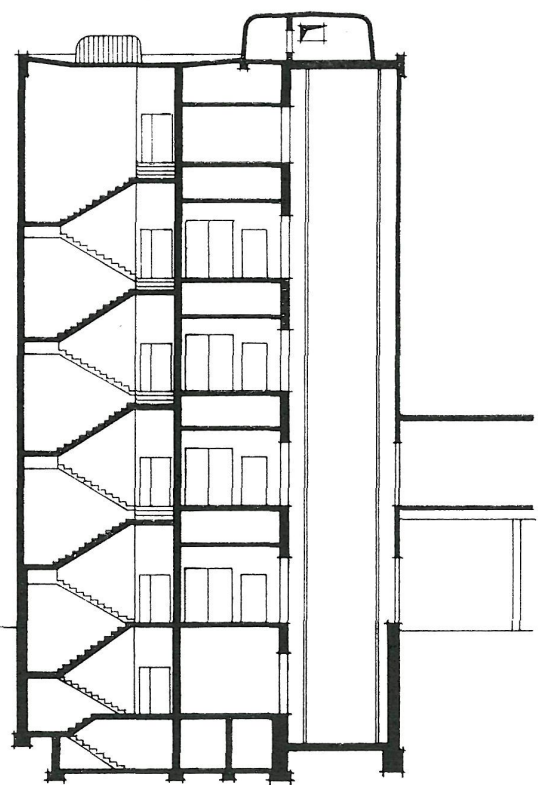

B-1B

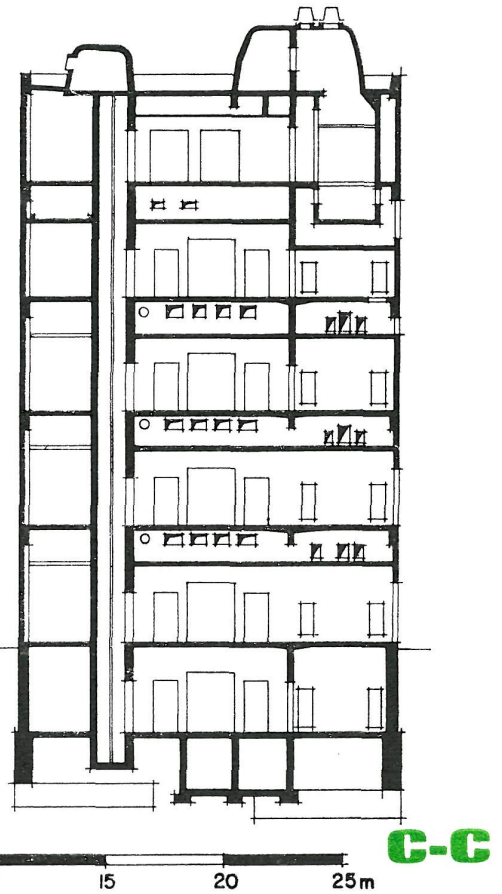

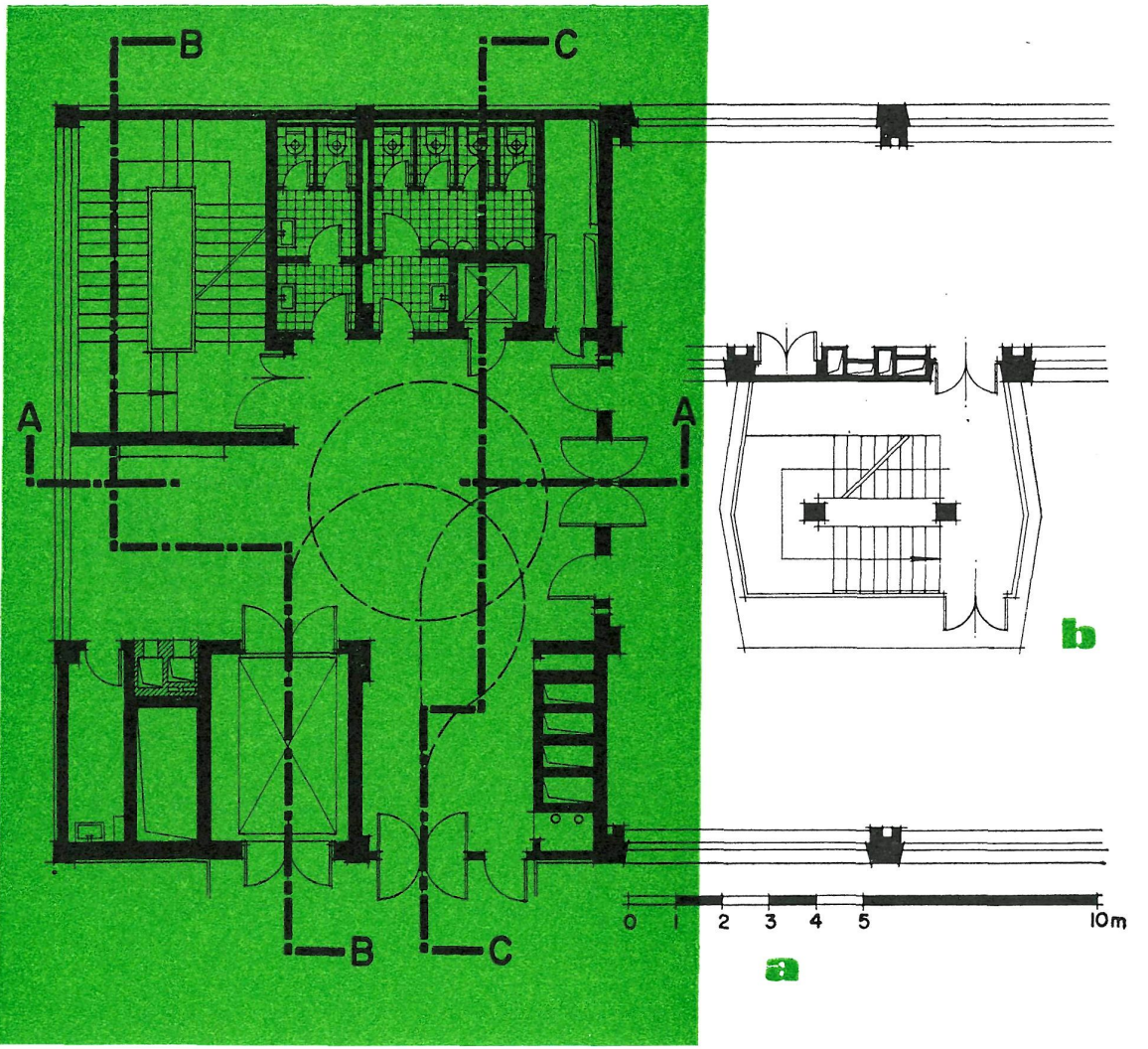

Pascerallem

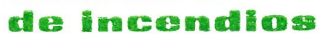

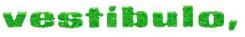

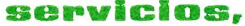

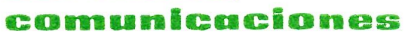
vertictiles 


\section{detalles de vontana}

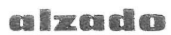
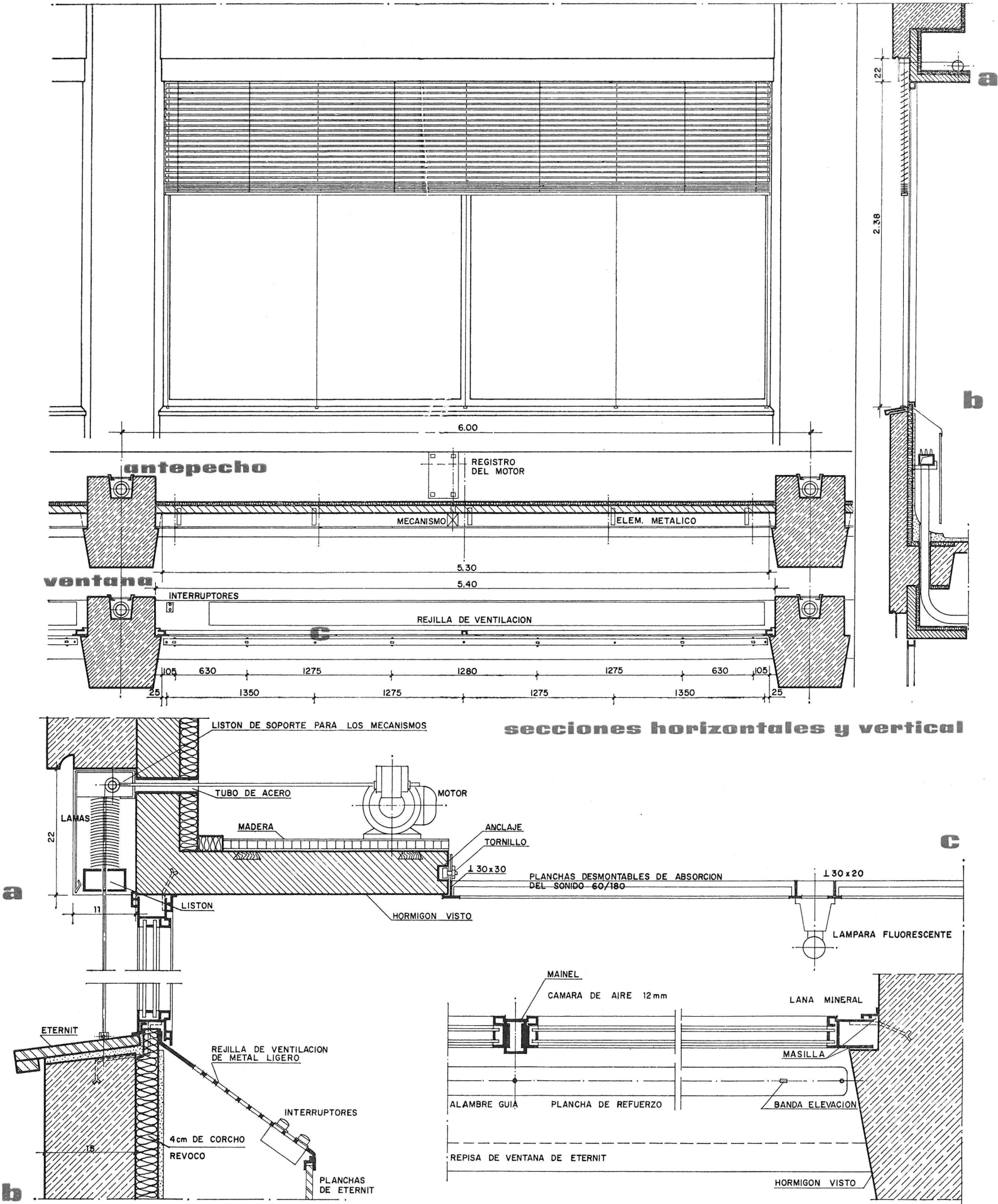

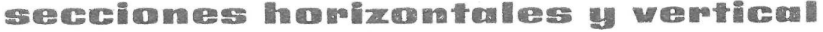
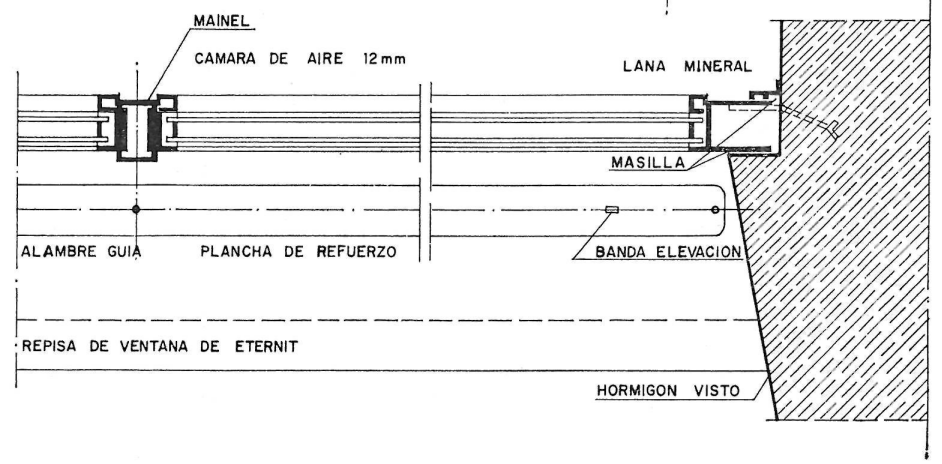
Las diferentes plantas están dedicadas a la fabricación, existiendo una segunda central de climatización para la segunda y tercera plantas sobre una terraza.

En cuanto a características constructivas: la estructura ha sido realizada a base de hormigón pretensado y está dividida en tres cuerpos mediante dos juntas de dilatación.

Los soportes aparecen resaltados de la línea de fachada, y acusados por su color oscuro; y los antepechos, de «hormigón visto», están pintados de blanco.

La cubierta está constituida por una estructura metálica ligera; su parte inferior presenta un revestimiento a base de placas de hormigón de piedra pómez. Y la cubierta consta de una capa de cartón y una lámina de material plástico. El canalón del tejado se calienta eléctricamente en invierno, con objeto de evitar la formación de hielo.

Adaptado por J. M. Rubio.

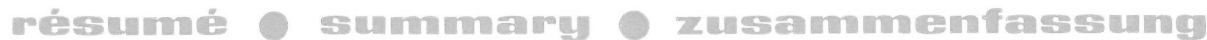

\section{Nouvel b́difice de l"usime Deckel à Munich = Allemagne}

Prof. Dr.-Ing. W. Henn, architecte; Dr.-Ing. J. Zeh, collaborateur

Destiné à la fabrication d'accessoires, cet édifice se compose d'un sous-sol, d'un rez-de-chaussée et de quatre étages.

Au sous-sol se trouvent une des centrales de climatisation, les vestiaires, les toilettes et les douches du personnel. Les autres étages sont consacrés à la fabrication. Finalement, la plus grande partie de la superficie du niveau supérieur est occupée par les bureaux et les locaux sociaux.

Cet édifice, doté d'un excellent éclairage et de conditionnement d'air, est clair, simple et fonctionnel.

\section{New building at the Deckel fuctory in Mumich, Gemmang}

Prof. Dr. Eng. W. Henn, architect; Dr. Eng. J. Zeh, collaborator

This firm manufactures accessories, and the new building has a basement, ground floor and four storeys above it. The basement houses the air conditioning plant, changing and wash rooms, and other services. The rest of the building is devoted to manufacturing, except for most of the top storey, which is taken up with office space and rooms for social activities.

The building is diaphanous, functional and has first rate air conditioning and lighting installations.

\section{Neues Gebueude der Fubrik Deckel in Muenchen - Deutschlemd}

Prof. Dr.-Ing. W. Henn, Architekt; Dr.-Ing. J. Zeh, Mitarbeiter

Dieses Gebaeude ist fuer die Fabrikation von Zubehoerteilen bestimmt und besteht aus: Kellergeschoss, Erdgeschoss und vier Stockwenken.

Im Kellergeschoss befindet sich eine der Klimazentralen, die Garderoben, Toiletten, Duschen fuer das Personal, usw. Die uebrigen Stockwerke dienen der Fabrikation; der groesste Teil der Flaeche des obersten Stockwerkes ist durch die Bueros und Gemeischaftsraeume belegt.

Das Gebaeude ist hell, einfach und zweckdienlich und besitzt eine vortreffliche Einrichtung und Beleutchtung. 